\title{
Summaries of Selected Papers Read at the Hyeres Meeting in France, January, 1984
}

\author{
Assessment of Different Types of Management at the Early Stage of \\ Spinal Cord Injury \\ By Professor A. Tricot and Dr E. Bobenrieth Centre for Traumatology and \\ Rehabilitation, Brugmann University Hospital, B - 1020 Brussels, Belgium
}

It is not easy to evaluate the different treatments adopted in the early stage of spinal cord injury; however, if two methods seem to be in opposition (spinal fixation by surgery on the one hand and orthopaedic treatment on the other), many factors intervening at the early stage can have an effect upon the eventual outcome. The evolution of a spinal cord lesion is characterised by three phases: (a) immediate phase; (b) acute phase; (c) long care hospital phase.

In the third phase the programmes of rehabilitation become a priority. Along with functional rehabilitation, social and professional resettlement problems assistance on site, the qualification of persons responsible for uplifting and transporting the patient, and the value, the rapidity and efficiency of the hospital emergency department.

During the acute phase (as during the immediate phase), prevention of the main complications have therapeutic priority, equal in importance to orthopaedic or neurosurgical therapy.

In the third phase the programmes of rehabilitation become a priority. Along with the functional rehabilitation, social and professional resettlement problems are closely associated.

To harmonize these three phases and to obtain a maximum of efficiency the spinal unit is ideal for the specialised care.

Not every vertebral fracture, with or without a spinal cord lesion, requires fixation by surgery. In the case of a spinal cord lesion the choice will depend on the orthopaedic and neurological aspects. The physician must act as a 'paraplegist' taking into account an analysis of the present situation, eventual complications and the long term results. The orthopaedic techniques, postural correction, manipulations, vertebral traction and corrective orthosis do not necessarily prolong the period of hospital care. The type and form of fusion or stabilising material is vast; and excessive material can cause a stiff spine, and muscular damage above the level of the lesion may result in post-operative pain. Indications for a laminectomy should be carefully considered. The danger of manipulation under anaesthesia and excessive traction is well known.

The improvement in our medical and scientific knowledge and treatment 
techniques do not mean the neglect of therapeutic methods which have proved their efficiency for over 40 years and which have enabled paralysed persons to resume their place in society.

\section{Spinal Cord Wounds by Projectiles}

By Dr B. Leriche, ${ }^{1}$ Dr R. Tronca, ${ }^{2}$ Dr M. Bonnevalle, ${ }^{3}$ Dr F. Lefebvre ${ }^{3}$ and Dr G. Lagrave ${ }^{4}$

${ }^{1}$ Neurochirurgien de hopitaux des Armces, ${ }^{2}$ Specialiste de Reeducation Fonctionnelle, ${ }^{3}$ Interne de hopitaux en chirurgie and ${ }^{4}$ Chirurigien de hopitaux des Armces.

The rarity of spinal cord projectile wounds explains the absence of specific therapy: the only retrospective studies concern the experience of armed conflicts.

In civilian practice, the wounded man will most certainly be referred to a neurosurgical hospital. Knowledge of the type of projectile and of the anatomical lesion resulting are essential to define the best specific therapy. The lesions are the result of projectile contusion and of the distant vascular lesions, resulting from the effect of blast; even the temperature of the projectile is important. Experience proves that many secondary painful or spastic phenomen are induced by an inadequate initial treatment, leaving persistance of microabscesses, syringomyelia ... etc. In fact, it is a matter of great urgency to treat all spinal cord wounds.

If the projectile entry wound and track involves the spinal axis the operation must be similar to that for the treatment of a cranio-cerebral wound with the treatment of osseous, meningeal, muscular and cutaneous lesions. This may be executed in one or two stages according to proximity of a neurosurgical hospital. The osseous stabilisation by osteosynthesis is contra-indicated, but may be only exceptionally necessary. If the projectile entry wound is in front or lateral, the visceral lesion is the dominant feature: lumbar puncture, myelography, angiography, or the C.T. scan in particular instances will disclose the type and extent of the spinal cord wound and therefore the necessity for surgical treatment by a posterior approach.

Only where there is a normal lumbar puncture and myelography, a posterior surgical approach, in a neurosurgical environment is justified and can be complemented by osteosynthetic stabilisation.

\section{Early Management of Dorso-Lumbar Injuries}

By Dr Norval Watson

Lodge Moor Hospital, Sheffield, U.K.

The early management of dorso-lumbar spinal injuries has always been controversial. Guttmann and his disciples advocated conservative methods. In the U.S.A. laminectomy and fixation have been popular.

In the early years of the Sheffield Unit, many miners with this injury were treated with open reduction and fixation. Now the Unit is much more selective, treating about 30 per cent of dorso-lumbar injuries surgically.

Indications for surgery are (1) Instability with gross displacement, (2) Pain, and (3) Lumbar segmental sparing. 
Clinical examination will reveal whether the lesion is complete or incomplete. If incomplete, and the bony injury is unstable, fixation is needed to protect the lumbar roots. If complete, then fixation is indicated only for pain from a badly displaced fracture.

Radiological examination will reveal whether the lesion is stable or unstable. Careful assessment will differentiate burst type injuries, which are mostly stable, unless gross, from fracture-dislocations, which are always unstable, and are recognised by rupture of the interspinous ligament revealing a gap and loss of alignment of the spinous processes, fracture or dislocation of the facets, and a slice fracture of the vertebral body, where the disc is carried forward with the body above.

A rational decision can then be taken, on clinical and radiological evidence, whether to treat conservatively or surgically.

All stable fractures are treated conservatively. All grossly displaced fractures are treated surgically, even if there is complete paraplegia.

Where the unstable fracture-dislocation has only minor displacement, surgery will depend on whether the lesion is complete or incomplete. If complete, treatment should be conservative. If incomplete surgery is advisable.

In operative cases, laminectomy is not performed, but the facets are manipulated into their normal position, and then a metal plate is fixed to the spinous processes. A Zadik clamp is used for dislocated facets, and Meurig-Williams plates where the facets are fractured. Harrington or Luque Rods are applicable in severe burst injuries.

Whether the treatment is conservative or surgical all patients are nursed using the same log-rolling method, using a special lumbar pillow, which fills the lordotic curve, supports and extends the fracture site, and is used to turn the patient. 